\title{
Design and development of a project-based embedded system laboratory using PIC 18F25K20
}

\author{
Rajeswari Cherukuri ${ }^{1}$, Raghavendra Rao Kanchi ${ }^{2, *}$ \\ ${ }^{1}$ Research Student, VLSI \& Embedded System Laboratory, Sri Krishnadevaraya University, Anantapuramu, 515003, INDIA \\ ${ }^{2}$ Chairman, BOS in Physics, VLSI \& Embedded System Laboratory, Sri Krishnadevaraya University, Anantapuramu, 515003, INDIA
}

\section{Email address:}

kanchiraghavendrarao@gmail.com (R. R. Kanchi)

\section{To cite this article:}

Rajeswari Cherukuri, Raghavendra Rao Kanchi. Design and Development of a Project-Based Embedded System Laboratory Using PIC 18F25K20. American Journal of Embedded Systems and Applications. Vol. 2, No. 3, 2014, pp. 21-28. doi: 10.11648/j.ajesa.20140203.12

\begin{abstract}
In this paper we present experiments and projects which gives hands-on experience to the students who start working with microcontrollers initially. As PIC microcontrollers have become popular and are one of the best microcontrollers to start working with in an introductory embedded system laboratory, we have chosen PIC 18 F25K20 microcontroller available on the market in a palm size module by name: AMICUS. The exercises described in this paper can be introduced as a one-third semester laboratory course. The AMICUS module is inexpensive and can be used to perform basic and project-based exercises. It is well suited for an under graduate engineering or science course, with electronics, computer science as major. If it is a introductory laboratory course on microcontrollers, it gives an opportunity for the student to use his previous knowledge on basic electronic circuits, digital system design, computer architecture and software development. This paper is not only useful for academics but also useful for amateur electronics hobbyist.
\end{abstract}

Keywords: Embedded System Laboratory, PIC 18F25K20, Project-Based Experiments

\section{Introduction}

We have communicated a couple of papers $[1,2]$ to this journal, describing the methodology to get acquainted with different advanced microcontrollers, which paves path for designing an embedded system laboratory. Indeed it is not only useful for academics, but also for amateur electronics hobbyist to learn using microcontrollers, which is the heart of the embedded system design. We received appreciation mails on these papers. Continuing in this direction, we have chosen designing with PIC microcontrollers which are widely used in industry, robotics and entertainment.

The technological advancement in the field of system-on-chip (SOC) has lead to the design and development of embedded systems. Further, the proliferation of such embedded systems in diversified fields has already begun. Embedded systems are widely used in cellular phones, digital cameras, home appliances, human healthcare, structure health monitoring systems, space applications up to ubiquitous computing. Looking from this perspective, it is the need of the hour to bring out young engineers who can take up challenging jobs in industry.

Looking from the industrial perspective, the outgoing student should have been exposed to team work, reasonably complex projects, in addition to the subject knowledge. This calls for practical and application-oriented training in education, with cooperative problem solving. If the course work satisfies all these aspects, the student can learn faster in the presence of team work and develop leadership qualities.

Research work was also carried out in different fields using PIC18F25K20 [3-7]. Keeping these facts in view point, we have developed more than a dozen of interfacing experiments using microchip low-power mixed signal controller PIC 18F25K20. Of course, microcontrollers with advanced architecture are also available. Our aim is to start humbly, with a simple microcontroller and then move to complex architectures.

In this paper, we used Amicus 18 development board; it was purchased from NSK Electronics, Bangalore [8]. Amicus 18 is an embedded system platform based on a simple open hardware design for a single-board microcontroller, with embedded I/O support and a standard programming language. The programming language is a version of Crownhill's proton BASIC. Amicus 18 is used to 
develop stand-alone interactive objects or it can be connected to a host computer. An Amicus 18 board consists of an 8-bit microchip PIC microcontroller: 18F25K20, with complementary components to facilitate programming and incorporation in to other circuits. The board includes both $5 \mathrm{~V}$ and $3 \mathrm{~V}$ linear regulators and a $16 \mathrm{MHz}$ crystal oscillator, thus allowing 16-MIPS operation. The Amicus 18's microcontroller $(18 \mathrm{~F} 25 \mathrm{~K} 20)$ is also pre-programmed with a boot-loader that simplifies uploading of programs to the on-chip flash memory compared to other devices that typically need an external chip programmer; Amicus 18 can be programmed via a USB cable that also serves as a means of power and communication to the board.

The Amicus 18 board is extremely easy to use; in fact, no previous microcontroller's experience is required in order to get the first project started up and running. Figure 1 shows the Amicus 18 development board.

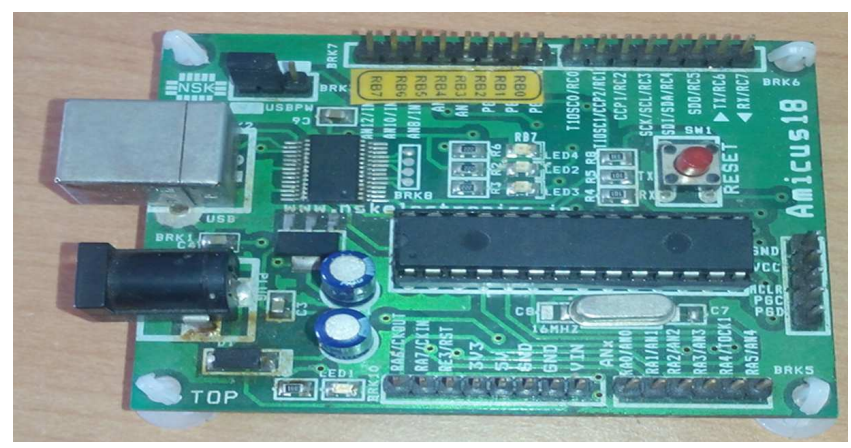

Fig. 1. Amicus 18 Development Board.

The experiments presented in this paper start with LED interfacing and goes up to the projects with interfacing of sensors. The systematic approach of learn-while-doing increase the confidence in students. Further, majority of the components used in the present work are off the shelf or low-cost, hence students can purchase on their own [9].

The experiments explained here, can also be useful for fresh research and graduate students who wants to work in the fields of instrumentation and electronics.

PIC 18F25K20 microcontroller is mixed signal controller that posses high processing power and sufficient on-chip memory. The hardware designed for experiments consume less power, and hence can be operated using battery. Further, it also occupies less space and function properly in real-time. These are the best qualifications for embedded system design.

This paper is organized as: Section-2 gives brief description of the architectural details of PIC 18F25K20 microchip mixed signal controller and programming procedure of the controller using proton BASIC. Section-3 gives the details of hardware and software, developed in the present study. Conclusions and scope for future work are included in Section 4.

\section{Architecture and Programming Procedure using Proton BASIC}

\subsection{Architecture of PIC 18F25K20}

The PIC 18F25K20 is an 8-bit mixed signal controller from Microchip, and is one of the most widely used microcontroller in a number of embedded system applications such as automobiles, industrial control etc. PIC 18F25K20 microcontroller offers low power consumption. It is this microcontroller used on the Amicus 18 board has 32768 bytes of flash memory, 1536 bytes of RAM, and operates at $64 \mathrm{MHz}$.

It has different power management modes, like: run, idle, and sleep, with two speed oscillation start up. It has four different timers, called Timer 0 , Timer 1, Timer 2, and Timer 3 , and also supports a sleep and watchdog timer. On utilizing these timers, the sensor node can be kept working for a longer period of time [10]. Other important features are:

$\rightarrow \quad$ It has high performance RISC CPU up to 16-MIPS operation.

$\rightarrow$ It has flexible oscillator structure with $16 \mathrm{MHz}$ oscillator.

The on-chip peripheral components of the PIC 18F25K20 include: 12 10-bit Analog-to-Digital converter channels, 2-Analog comparator module, Two 10-bit PWM outputs, A USART, SPI, I2C and 4-timers [11]. It supports the operating system such as windows. Figure 2 shows the internal architecture of PIC 18F25K20

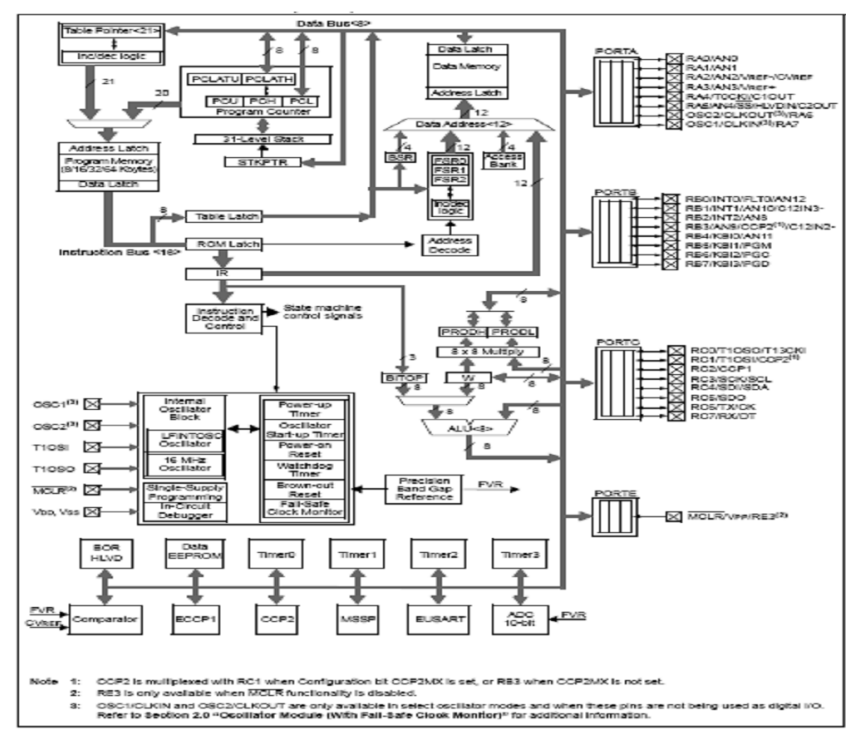

Fig. 2. Internal Architecture of PIC 18F25K20.

\subsection{Programming Procedure using Proton BASIC with Amicus IDE}

The proton Amicus 18 IDE is a professional and powerful Integrated Development Environment (IDE) designed specifically for the Amicus 18 development board. It is designed to accelerate product development in a comfortable user friendly environment without compromising 
performance, flexibility or control. The proton BASIC compiler is free compiler specially adapted for the Amicus 18 development platform. The compiler has no restrictions or time limits and is fully functional [12].

Programming steps involved in the development of a project are given below:

Install the Amicus 18 BASIC compiler which is around $50 \mathrm{Mb}$ in size. Once installed, clicking on the Amicus 18 IDE, Icon is brought to the main program IDE. This is to write and edit the BASIC source code and start the compile process. Now it is ready to generate the HEX code for transferring to the on chip microcontroller PIC 18F25K20.

The Amicus IDE is divided into four main parts.

1. The menu and tool bar will be at the top. 2. Below the toolbar there is code explorer window at the left side. As we start to write the program, variables, labels and other definitions will appear on this window. 3 . On right side there is large main code entry window where we enter the program source text. 4 . There is a bottom section where status of information is shown.

Once entered the text, it is always required to save program. By Pressing the "save" button from the toolbar, the IDE asks where would you like to store the file. Then store the files at the default location (or) any other place. Do not place them at the root of a drive. When the compile process starts it creates all sorts of additional files that soon clutter the place up.

Give the file name, for example program 1 and press the save button in the dialogue box.

Next locate the "compile" button on the IDE toolbar (left hand side) and click it. Amicus will perform a sequence of activities including checking the text for obvious errors and attempting to turn the statements to in PIC assembler (.ASM file).

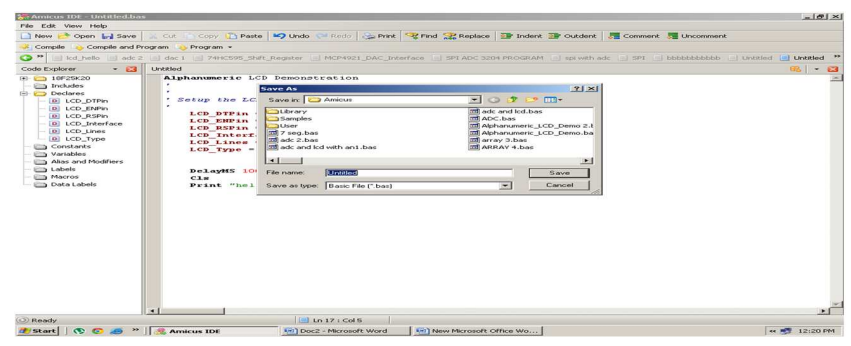

Fig. 3. Screen shot for save the project.

If the typed program is correct then a few seconds later the bottom of the IDE screen change to something like this:

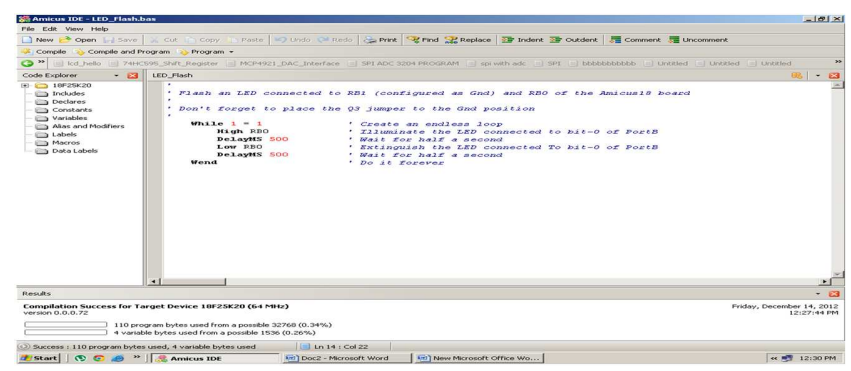

Fig. 4. Screen shot for compilation.
If the program has mistakes, the compiler will attempt to tell where they are.

Once finished writing the program, by hitting compile+program and the Amicus IDE will promtly have the PIC flashed.

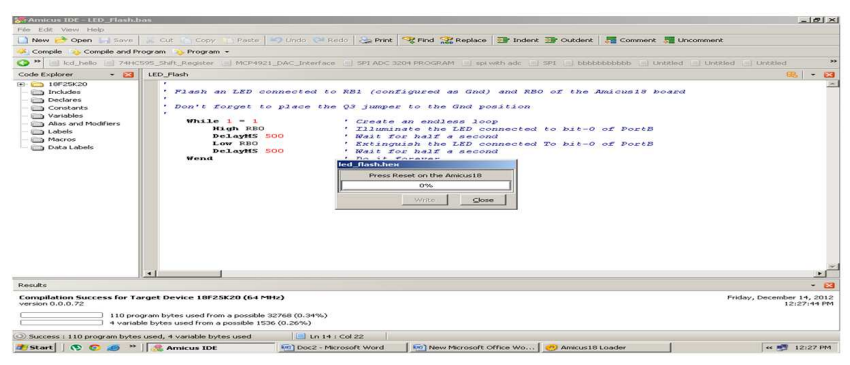

Fig 5. Screen shot for dumping the program in to the Amicus 18.

\section{Details of Individual Experiments}

The total experiments developed and described in this paper are divided into three modules A,B and C. The first module explains the basic input/output interfacing. Module B corresponds to the experiments for exploitation of the on-chip peripherals. Sensor interfacing with Amicus 18 is described in module $\mathrm{C}$.

\section{Module A: Basic I/O Interfacing}

This module contains interfacing experiments, relating to I/O programming. Interfacing exercises starts with LED blinking program and covers push button, stepper motor and LCD.

\section{Ex.A 1: LED Blinking}

The first experiment is LED blinking/ toggling. This is the basic experiment in embedded systems. Light Emitting Diode (LED) is the most commonly used component, usually for displaying the digital status of I/O pins.

To get acquainted with GPIO pins, LEDs are connected to port pins RB0 to RB7 of PORT B with current limiting resistors $(220 \Omega)$, mounted on a bread board. The program is developed using proton BASIC .

The software is developed based on the following algorithm:

Step 1. Start

Step 2. Create a variable to hold the state of PORT B.

Step 3. Make Port B output low.

Step 4. Transfer the variable value to Port B.

Step 5. Shift a bit left one position.

Step 6. Shift a bit right one positin.

Step 7. Do it forever.

Figure A1 shows the photograph of the experiment 


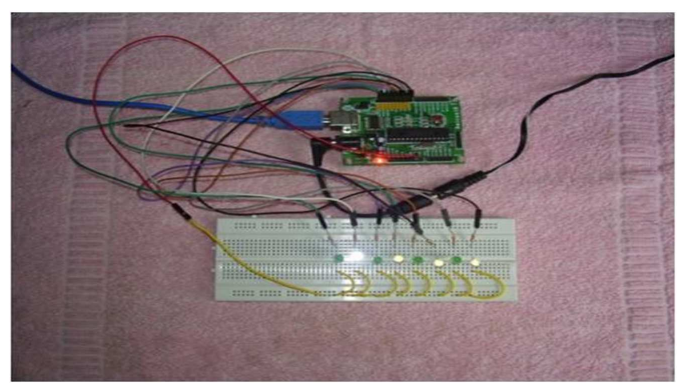

Fig. A1. Amicus 18 interfaced with LEDs

\section{Ex. A 2: Push button interface}

The main aim of the experiment is to debounce a mechanical switch by software while counting the number of times the button is pressed. The status of the pushbutton after debouncing is observed using LED connected to RB0 while the push button is connected to RB1. All these components are placed on the bread board and connected to Amicus 18 board as shown in figure A2.

The software is developed based on the following algorithm:

Step 1. Start

Step 2. Define a variable to hold the switch pressing.

Step 3. Make Port B as output.

Step 4. Make the switch pin input

Step 5. Debounce the switch.

Step 6. Do it forever.

Figure A2 shows the photograph of the experiment

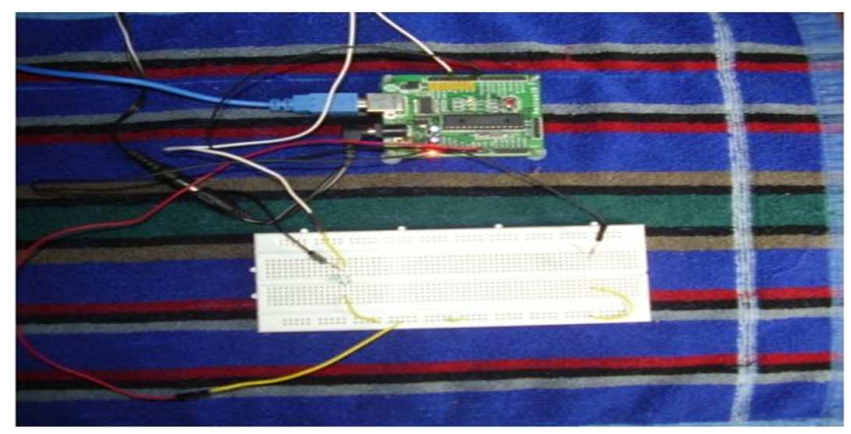

Fig. A2. Amicus 18 interfaced with push button

\section{Ex.A 3: Seven segment Display}

In this experiment RB0 to RB7 of PortB pins are used for interfacing a seven segment display. Common anode type seven segment display, current limiting resistors, bread board and connecting wires are required to construct the hardware.

The software is developed based on the following algorithm

Step 1. Start

Step 2. Define a variable to hold the data to be displayed.

Step 3. Make Port B as output.

Step4. Display variable data on Port B.

Step5. Define delay

Step 6. Repeat forever

Figure A3 shows the photograph of the experiment

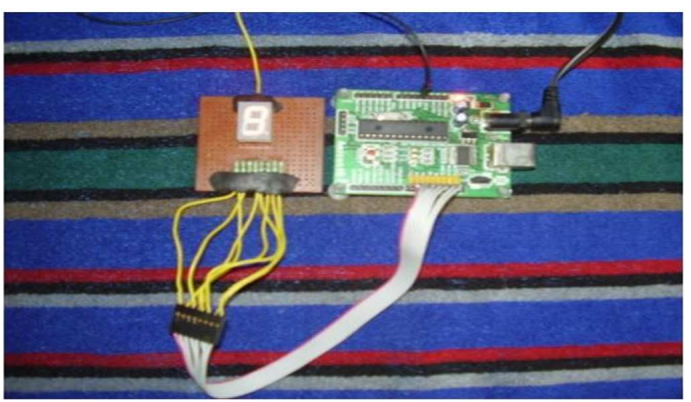

Fig. A3. Amicus 18 interfaced with seven segment, Hex value ' 8 ' is displayed on the seven segment display

\section{Ex. A 4: Stepper Motor Interfacing Experiment}

In this experiment the stepper motor is interfaced with Amicus 18 board using Port B pins RB0 to RB 7. ULN 2003 is used for driving the motor. Because the controller can't give the sufficient current to output port to driven the motor. Required components are stepper motor, IC ULN 2003, Connecting wires and bread board.

The stepper motor is rotated in steps continuously, by developing the software basing on the following algorithm:

Step 1. Start

Step2. Initialize Port B as output port.

Step 3. Put $88 \mathrm{H}$ in B register.

Step 4. Call delay.

Step 5. Rotate B register right.

Step 6. Stop

Figure A4 shows the photograph of the experiment

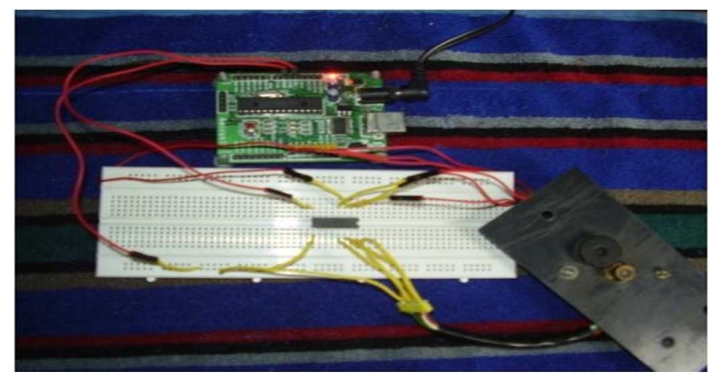

Fig. A4. Amicus 18 interfaced with stepper motor

\section{Ex. A 5: LCD Interfacing Experiment}

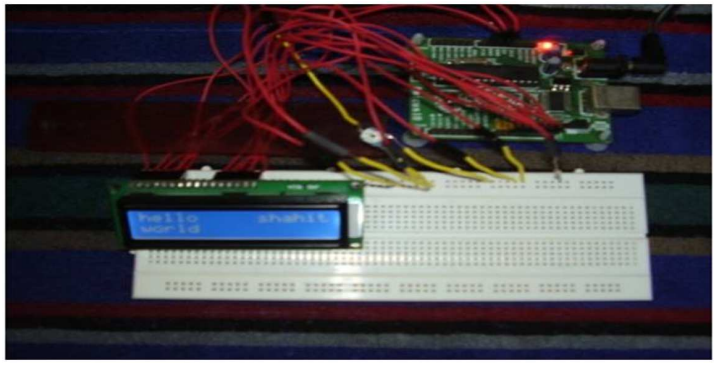

Fig. A5. Amicus 18 interfaced with LCD

A $16 \mathrm{X} 2$ LCD module is used in this experiment. LCD module is used in 4-bit mode. RB4 to RB 7 of Port B pins are used for sending the data, RB2 and RB3 are used as control pins, between microcontroller and LCD module. 
LCD module, $10 \mathrm{~K} \Omega$ pot, bread board and connecting wires are required for hardware.

The LCD display software is developed basing on the following algorithm:

Step 1. Start

Step 2. Initialize the LCD with 2 lines.

Step 3. Clear LCD.

Step 4. Display “ HELLO” on 1 line.
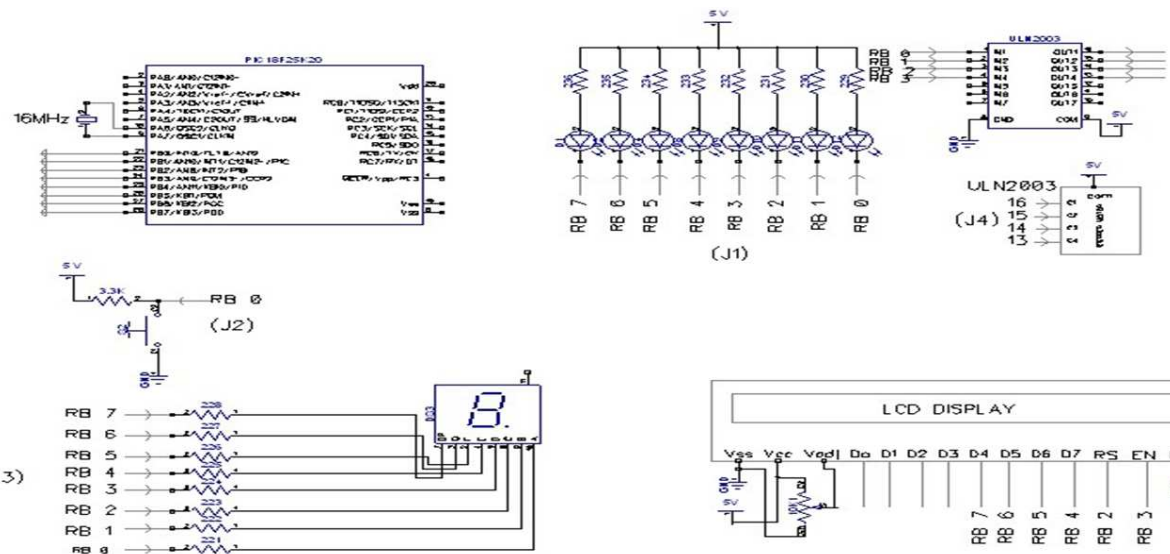

Step 5. Make delay.

Step 6. Display "WORLD" on 2 line.

Step 7. Make delay.

Step 8. Stop

Figure A5shows the photograph of the experiment

The total hardware circuits for Module A are shown in figure A6.

Fig. A6. Total hardware schematics for experiments A1 to A5

\section{Module B: Interfacing Experiments to Exploit the on-Chip Peripherals}

One of the interesting features of PIC $18 \mathrm{~F} 25 \mathrm{~K} 20$ is that each pin of PIC 18F25K20 can perform up to three different functions, which are selectable. This module gives the information about interfacing of the on-chip peripherals like Analog to Digital converter (ADC), DAC using ADC, Pulse Width Modulation (PWM), Timers/Counters, Analog comparator and SPI (Serial Peripheral Interface).

\section{Ex. B1: Measurement of Analog Voltage using on-Chip $A D C$}

PIC 18F25K20 has a 10-bit on-chip analog-to-digital converter (ADC). Amicus 18 has 4-Analog input channels. In this ANO (Analog channel 0) is used for converting analog data and corresponding digital data be displayed on LCD.

The software is developed based on the following algorithm:

Step 1. Start

Step 2. Load the ADC routines in to the program.

Step 3. Define 16-bit unsigned variable to hold the result of the ADC.

Step 4. Define an infinite loop.

Step 5. Take a reading from AN0 (ADC channel 0).

Step 6. Initialize the LCD with 2 lines.

Step 7. Clear LCD.

Step 8. Transmit the result to LCD.

Step 9. Call delay.

Step 10. Repeat.

Figure B 1 shows the photograph of the experiment

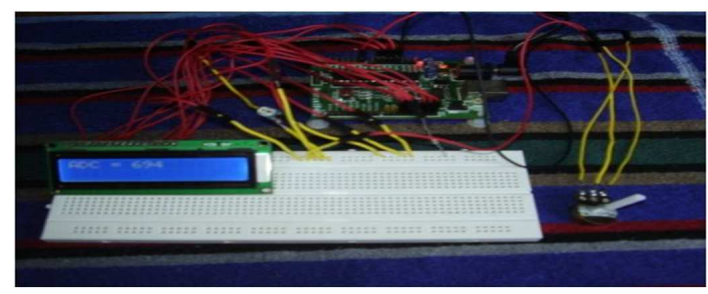

Fig . B1. Photograph of Analog input voltage

Ex. B2: Measurement of Digital Voltage Using On-Chip ADC and On-Chip Analog Comparator

The software is developed based on the following algorithm:

Step 1. Start

Step 2. Define a variable to hold the data from counter.

Step 3. Define another variable to hold the $\$ F 0$ data.

Step 4. Enable the Port B.

Step 5. Load the Analog comparator routines in to the program.

Step 6. Open the comp1.

Step 7. Enable the Port A.4.

Step 7. Enable the Port A.4.

Step 8. Connect LED to Port A.4.

Step 9. Enable the suitable an-comp registers.

Step 10. Load the data to Port B.

Step 11. Call delay.

Step 12. If PORT A.4=1 then glow LED.

Step 13. Call delay.

Step 14. Else low LED.

Step 15. End

Figure B 2 shows the photograph of the experiment 


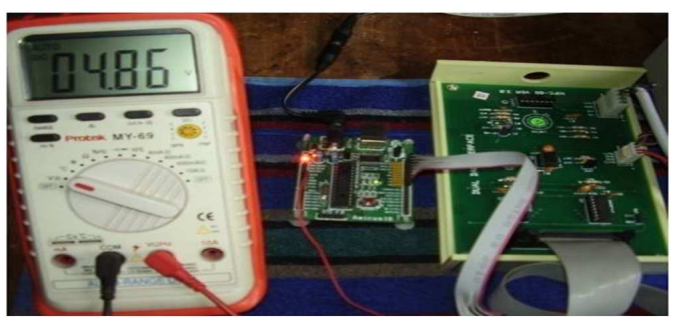

Fig. B2. Photograph of Analog output voltage

\section{Ex.B3: Realization of PWM using LED}

Amicus 18 development board has two PWM channels. Here CCP 1 channel is used for PWM. The pulse width modulation out at pin $\mathrm{RC} 2$ is realized using LED. Components required: LED, resistor and connecting wires. The software is developed based on the following algorithm:

Step 1. Start

Step 2. Load the 10-bit PWM macros into the program.

Step 3. Define the variables to hold the duty cycle of the PWM pulses.

Step 4. Enable and configure CCP 1 peripheral.

Step 5. Create an infinite loop.

Step 6. Cycle the full range of 10-bits.

Step 7. PWM on CCP 1.

Step 8. Call delay

Step 9. Cycle the full range of 10-bits

Step 10. PWM on CCP 1.

Step 11. Call delay.

Step 12. Close the loop.

\section{Ex. B4: Frequency Measurement System}

Timer0 is 16-bit timer in PIC 18F25K20. Timer0 input pin $\mathrm{RC} 0$ is used for the measurement of frequency of the input pulse. Here the input frequency is generated from frequency generator. Timer0 is set in counter mode. On falling edge of the pulse the timer resister is stored the data. TimerOL is stored the low byte data and TimerOH is stored the high byte data. The stored data will send to PC through the serial port of Amicus 18. Required components are: frequency generator and connecting wires.

The software is developed based on the following algorithm:

Step 1. Start

Step 2. Load the timer macros into the program.

Step 3. Enable the suitable timer registers.

Step 4. Call delay.

Step 5. Close the timer register.

Step 6. Display the counter value on serial terminal.

Ex B5: SPI (Serial Peripheral Interface)

In AMICUS 18 board it has one SPI (Serial Peripheral Interface). In this RC2 to RC5 of Port $\mathrm{C}$ pins are used to SPI. The SPI mode allows 8-bit of data simultaneously transmitted and receive. Here we observed the serial transferred data on LCD by varying the POT. Required

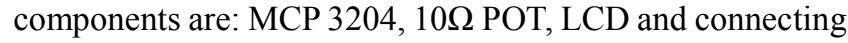
wires.

The software is developed based on the following algorithm:

Step 1. Start

Step 2. Load SPI macros in to the program.

Step 3. Initialize the LCD with 2 lines.

Step 4. Initialize the Port C for MCP 3204.

Step 5. Define variables to hold the address of the MCP 3204.

Step6. Define variables to hold the data read from SPI.

Step 7. Clear LCD.

Step8. Open the SPI.

Step9. Enable the PORTC.

Step 10. Clear the CS of MCP 3204.

Step 11. Write address of MCP 3204 to SPI.

Step 12. Define an infinite loop.

Step 13. Read SPI and STORE it on variable.

Step 14. Call delay.

Step 15. Print data on 1 line of LCD.

Step 16. Set the CS of MCP 3204.

Step 17. Call delay.

Step 18. End
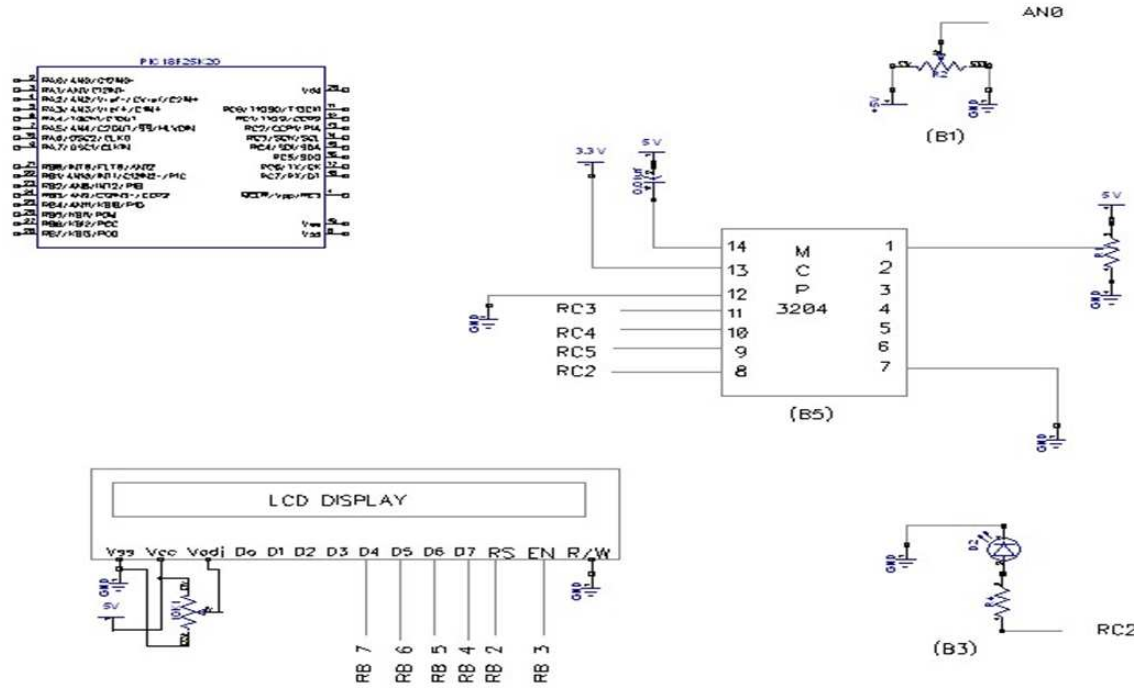

Fig.8. Shows Hardware Schematic of Peripheral interfacing 


\section{Module C: Project Based Embedded Application with Sensor Interfacing Experiment}

\section{Ex.C1: HSM-20G Humidity and Temperature} Measurement Sensor Interfacing with PIC 18 F25K20

The HSM 20G module is interfaced with PIC 18F25K20 microcontroller. Analog input channels of AN0 and AN1 are used to measure the Relative humidity present in air and Temperature at room. Components required: HSM 20G sensor, LCD and connecting wires.

The software is developed based on the following algorithm:

Step 1. Start

Step 2. Initialize the LCD with 2 lines.

Step 3. Load the ADC macros in to the program.

Step 4. Define the suitable variables to hold the humidity and temperature data.

Step 5. Open the ADC with two channels.

Step6. Call delay.

Step 7. Clear the LCD.

Step 8. Define the infinite loop.

Step 9. Select the channel 1 of ADC.
Step 10. Read the temperature data from channel 1 of ADC.

Step 11. Print the data on LCD in 1 line.

Step 12. Call delay.

Step 13. Select the channel 0 of ADC.

Step 14. Read the humidity data from channel 0 of ADC.

Step 15. Print the data on LCD in 2 line.

Step 16. Call delay.

Step 17. Loop forever

Figure $\mathrm{C} 1$ shows the photograph of the experiment

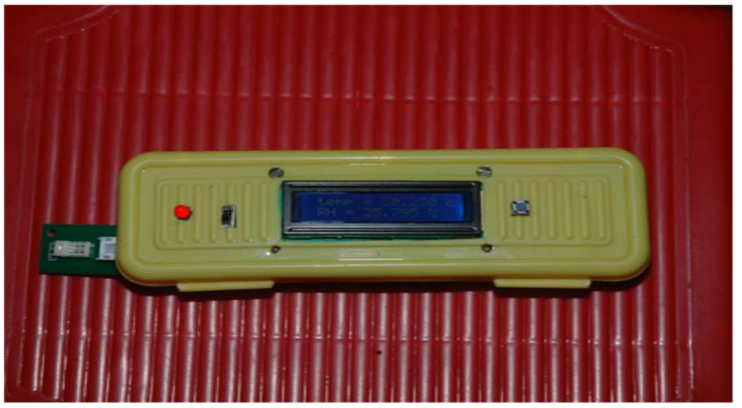

Fig . C1. Photograph of HSM 20 G (humidity and temperature measurement sensor) interfaced with PIC 18F25K20 microcontroller
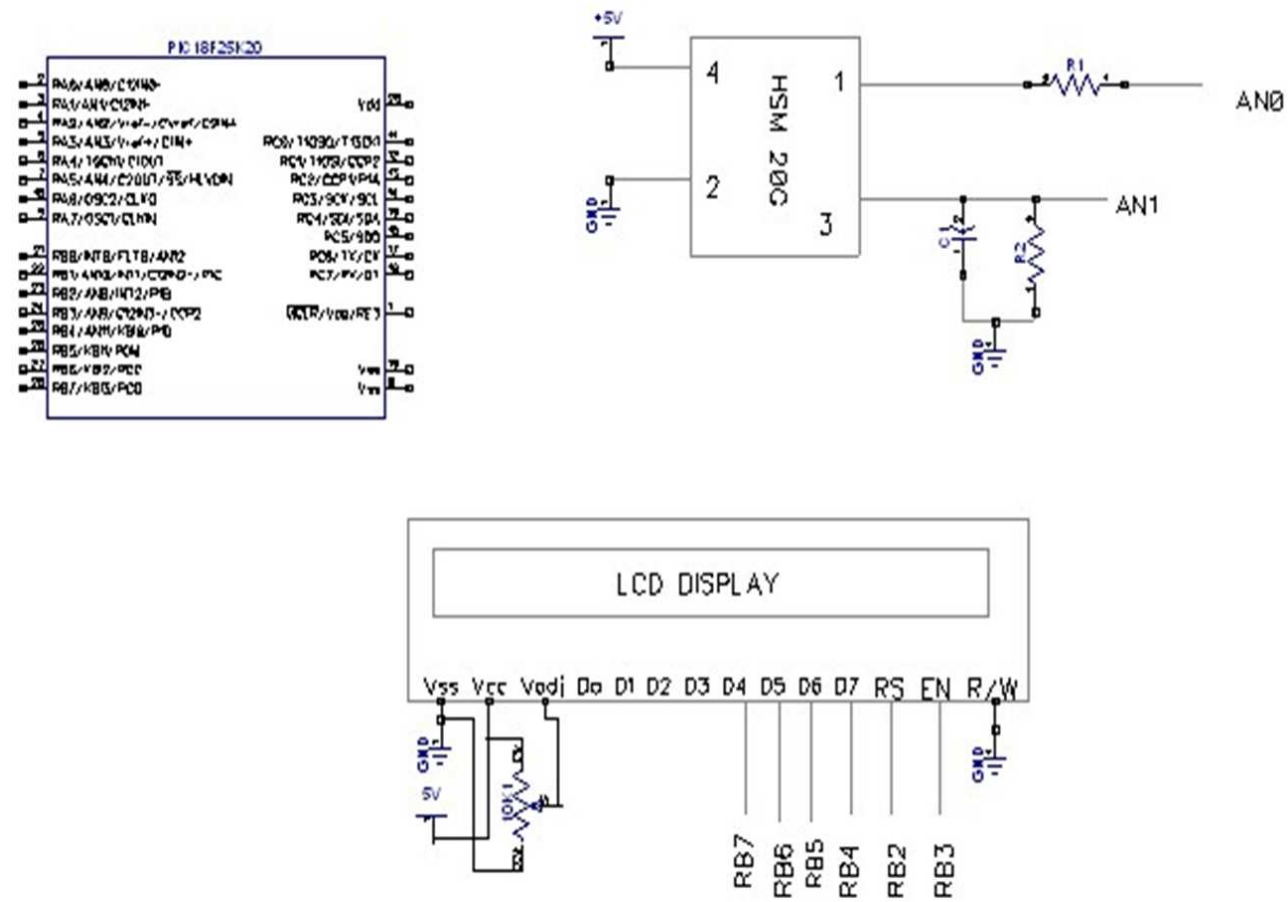

Fig. 9. Shows Hardware Schematic of HSM 20 G sensor

\section{Conclusion}

In this paper, bunch of experiments are designed and developed using Amicus 18 development board. These experiments can be introduced in a laboratory course as take-home experiments, because of its low cost. The hands-on experience a student gets by performing these experiments definitely gives a confidence to think and develop a complex embedded system, which is very much essential in the present scenario.

\section{Acknowledgement}

The facility created by the Department of Science and Technology (DST), New Delhi in sanctioning the FIST program (Phase-I) is acknowledged.

Software for the above programs can be obtained from authors on request. 


\section{References}

[1] Aruna Kommu, Raghavendra Rao Kanchi "Design and development of a project-based embedded system laboratory using LPC1768", American Journal of Embedded Systems and Applications. Vol.1,No.2, 2013,pp. 46-53.

[2] Naveen Kumar Uttarkar, Raghavendra Rao Kanchi. Design and Development of a Low-cost Embedded System Laboratory Using TI MSP430 Launchpad. American Journal of Embedded Systems and Applications. Vol.1, No.2,2013,pp.37-45.

[3] T. Hui Teo, Wee Tiong Tan, Pradeep K. Gopalakrishnan, Victor K.H.Phay, and $\mathrm{Ma} \mathrm{Su}$ M.M.Shwe "Wireless Healthcare Monitoring System for Home", World Academy of Science, Engineering and Technology vol:2 2008-06-23.

[4] "Development platform for Wireless Temperature Sensor".www. embeddedadventures.com.

[5] "Improved Energy Modelling of Wireless Personal Area Network" School of information sciences, Computer and Electrical Engineering Halmtad University.
[6] "High Resolution, Low Cost, Privacy Preserving Human Motion Tracking System via Passive Thermal Sensing" Department of Electrical Engineering and Computer Science. Massachusetts Institute of Technology. MIT Media Laboratory.

[7] \#.dspace.mit.edu/bitstream/handle/1721.1/702368984.pdf.

[8] www.nsk electronics.com.

[9] Wilfried Elmenreich, Christian Trodhandl and Bettina weiss, "Embedded system Home Experimentation",Proceedings of the 2003 IEEE International Conference on Microelectronic systems Education(MSE”03).

[10] "Improved Energy Modelling of Wireless Personal Area Network" School of information sciences, Computer and Electrical Engineering Halmtad University.

[11] www.Amicus.co.in

[12] www.crownhill.co.uk 